\title{
Passively biased resonantly enhanced silicon photonics modulator with high optical bandwidth
}

Sebastian Romero-García, Alvaro Moscoso-Mártir, Saeed Sharif Azadeh, Bin Shen, Jovana Nojic, et al.

Sebastian Romero-García, Alvaro Moscoso-Mártir, Saeed Sharif Azadeh, Bin Shen, Jovana Nojic, Florian Merget, Jeremy Witzens, "Passively biased resonantly enhanced silicon photonics modulator with high optical bandwidth," Proc. SPIE 10108, Silicon Photonics XII, 1010802 (13 March 2017); doi: $10.1117 / 12.2253845$

SPIE. Event: SPIE OPTO, 2017, San Francisco, California, United States 
Invited Paper

\title{
Passively Biased Resonantly Enhanced Silicon Photonics Modulator with High Optical Bandwidth
}

\author{
Sebastian Romero-García*, Alvaro Moscoso-Mártir, Saeed Sharif Azadeh, Bin Shen, Jovana Nojic, \\ Florian Merget, Jeremy Witzens \\ RWTH Aachen University, Institute of Integrated Photonics, Aachen, Germany;
}

\begin{abstract}
Ring resonator modulators reach high modulation efficiencies, are very compact and can be electrically driven as lumped elements. However, their limited optical bandwidth requires temperature stabilization, limiting their power efficiency. A novel ring assisted Mach-Zehnder modulator (MZM) aggressively reduces power consumption. Moreover, an integration scheme passively sets the $3 \mathrm{~dB}$ point during attachment of the input fiber relative to a multimode grating coupler used as the first splitter element of the interferometer. Straight phase shifters are replaced by arrays of highly overcoupled resonators maintaining a sufficiently high finesse and a substantial resonant enhancement while minimizing the excess losses at the resonator to waveguide junctions. A large resonance bandwidth compatible with thermal operation over $50{ }^{\circ} \mathrm{C}$ without dynamic compensation is obtained together with a factor larger than four in the reduction of power consumption relative to a conventional MZM.
\end{abstract}

Keywords: Optical modulators, integrated photonics, optoelectronics, silicon photonics, ring resonator modulators, MachZehnder modulators, grating couplers.

\section{INTRODUCTION}

Next generation transceivers for Datacom applications demand energy efficient and high optical bandwidth electro-optical modulators that also meet high density requirements and low manufacturing costs ${ }^{1-4}$. Silicon Photonics technology is one of the leading candidates to fulfill all these requirements by leveraging standard CMOS design and manufacturing. The mainstream configurations of Silicon Photonics modulators are twofold: Ring Resonator Modulators (RRM) ${ }^{5,6}$ and MachZehnder Modulators (MZM) $)^{3,7}$. RRMs leverage the resonance enhancement to reach high modulation efficiencies, are very compact and can be electrically driven as lumped elements. However, the resonant enhancement also limits the optical bandwidth. This limitation, together with the high thermo-optic coefficient of silicon, makes conventional RRMs fabricated in silicon-on-insulator technology very sensitive to temperature fluctuations, which leads to the requirement of an active resonance wavelength stabilization system ${ }^{4}$. The total power consumption of transceivers based on RRMs is mostly determined by the consumption of the active stabilization $\left(14 \mathrm{~mW}\right.$ for a $50{ }^{\circ} \mathrm{C}$ stabilization range in state-of-the-art configurations $^{8}$ ) that is significantly higher than the power required by the modulator driver (on the order of $0.8 \mathrm{~mW}$ at 10 $\mathrm{Gbps}^{8}$ ). On the other hand, MZMs relying on reverse biased PN junctions typically need long phase shifters or high drive voltages to reach high extinction. Furthermore, although a symmetric MZM configuration enables very large optical bandwidths, the compensation of fabrication asymmetries accumulating over long MZM arms requires a corrective phase tuner that increases both the power consumption and the operation complexity.

Here, we present novel MZM modulators that address these issues and aggressively reduce power consumption. First, an integration scheme passively sets the $3 \mathrm{~dB}$ point during attachment of the input fiber relative to a misalignment tolerant Multimode Grating Coupler (MMGC) also used as the splitter element of the interferometer ${ }^{9}$. This scheme avoids the necessity of corrective phase tuners and the associated power consumption. Second, the modulator configuration is based on a Resonantly-Enhanced MZM (RE-MZM) where the straight phase shifters of conventional MZMs are replaced by arrays of highly overcoupled resonators ${ }^{10-12}$. The challenge lies in maintaining a sufficiently high optical bandwidth and a substantial modulation enhancement while minimizing the excess losses at the junction between the bus waveguide and

* sromero@iph.rwth-aachen.de; phone +49 24180 20030; fax +49 24180 22212; iph.rwth-aachen.de 


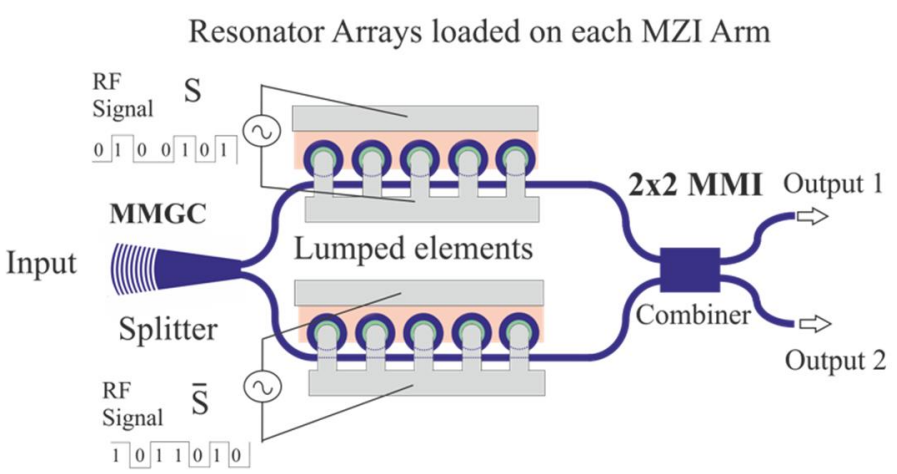

(a)

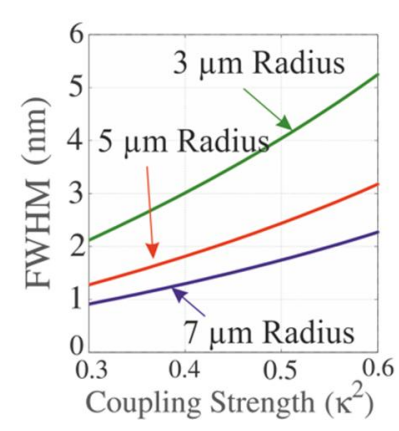

(b)

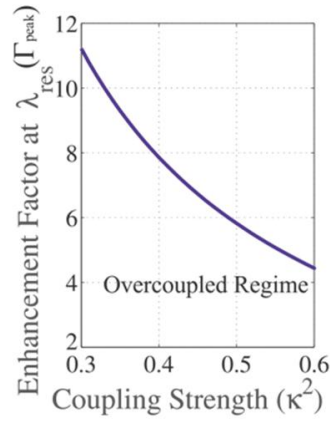

(c)

Figure 1. a) Schematic representation of the proposed MZM with Multimode Grating Coupler (MMGC) at the input and arrays of identical resonators loaded on each arm. b) Full-Width at Half-Maximum (FWHM) of the resonance (indicative of the optical bandwidth) and c) peak enhancement factor at the resonance wavelength $\left(\Gamma_{\text {peak }}\right)$, both shown as a function of the designed coupling strength $\left(\kappa^{2}\right)$.

the resonator. In this work, we show that by optimizing the resonator design it is possible to achieve a very compact cavity size and thus an acceptable finesse notwithstanding the low quality factor of the resonant phase shifter elements.

In section 2 we describe the working principle of a Mach-Zehnder modulator with resonantly enhanced phase shifters (REMZM) and a passive biasing scheme based on fiber integration with a multimode grating coupler. Section 3 is dedicated to the description of two active resonator designs for devices respectively based on lateral and vertical PIN junctions. For each of these we also present, in section 4 , the corresponding complete modulator design and expected performance. Finally, in this last section we also report experimental results for the modulator with lateral PN junction and passive biasing.

\section{DEVICE CONCEPT}

\subsection{Working principle}

For the implementation of the MZI, we selected a symmetric configuration based on a MMGC and 2 × 2 Multimode Interferometer (MMI) as splitter and combiner elements, respectively (see Figure 1(a)). The power levels at the outputs of the MZI combiner (assuming ideal splitter and combiner) vary according to:

$$
\begin{aligned}
& \mathrm{P}_{\text {output } 1}=\mathrm{A} \cdot \cos ^{2}\left(\left(\Delta \varphi_{R F}+\Delta \varphi_{0}+\pi / 2\right) / 2\right) \\
& \mathrm{P}_{\text {output2 }}=\mathrm{A} \cdot \sin ^{2}\left(\left(\Delta \varphi_{R F}+\Delta \varphi_{0}+\pi / 2\right) / 2\right)
\end{aligned}
$$

where $\mathrm{A}$ is the optical power attenuation in the splitter, combiner and interferometer arms, $\Delta \varphi_{\mathrm{RF}}$ is the phase shift difference at the input of the combiner due to the applied RF signal and $\Delta \varphi_{0}$ corresponds to the phase deviation from the quadrature point in the absence of RF driving voltage. In the selected configuration $\Delta \varphi_{0}$ results from fabrication asymmetries between the MZI arms. We propose to compensate for these asymmetries by proper alignment and attachment of the fiber on the MMGC (details in section 2.2).

In the proposed device, the straight phase shifters of conventional MZMs used for inducing the modulation phase $\Delta \varphi_{\mathrm{RF}}$ are replaced by arrays of identical and collectively driven RRMs, optically coupled to the interferometer arms. In order to achieve a wide optical bandwidth, or, equivalently, a large temperature range of operation, without the necessity of active resonance wavelength stabilization, the RRMs are overcoupled with a high coupling strength $\left(\kappa^{2}\right)$ between the bus waveguide and the cavity. In this regime, the Full-Width at Half Maximum (FWHM) of the resonance can be approximated by:

$$
\mathrm{FWHM} \approx-\frac{\lambda^{2} \cdot \log _{n}\left(t^{2}\right)}{(2 \pi)^{2} n_{g} \cdot R}
$$


with $\lambda$ the operating wavelength, $\mathrm{n}_{\mathrm{g}}$ the group index, $\mathrm{R}$ the resonator radius and $t^{2}$ the transmission coefficient in the coupling region of the resonator. Figure 1(b) shows the increase in the resonance FWHM with both increasing coupling strength (higher $\kappa^{2}$ ) and decreasing resonator radius. For this calculation, we have assumed that the excess loss in the coupling section is negligible and therefore $\kappa^{2}=1-t^{2}$. We have targeted resonator designs with a FWHM around $2 \mathrm{~nm}$. Since the typical drift of the resonance wavelength with temperature in silicon-on-insulator resonators is on the order of $72 \mathrm{pm} /{ }^{\circ} \mathrm{C}$, the selected $\mathrm{FWHM}$ corresponds to a thermal range on the order of $30^{\circ} \mathrm{C}$.

In the proposed MZM, each RRM is used as a phase modulator and exhibits a transfer function given by:

$$
\begin{aligned}
& \frac{E_{\text {out }}}{E_{\text {in }}}=\frac{t-\exp \left([\mathrm{j} \beta-\alpha / 2] \mathrm{L}_{\mathrm{PS}}\right)}{1-t \cdot \exp \left([\mathrm{j} \beta-\alpha / 2] \mathrm{L}_{\mathrm{PS}}\right)} \approx \frac{t-1-[\mathrm{j} \beta-\alpha / 2] \mathrm{L}_{\mathrm{PS}}}{1-t \cdot\left(1+[\mathrm{j} \beta-\alpha / 2] \mathrm{L}_{\mathrm{PS}}\right)} \approx-1-\frac{t+1}{1-t}[\mathrm{j} \beta-\alpha / 2] \mathrm{L}_{\mathrm{PS}} \approx \\
& \approx-1+\frac{4}{\log _{n}\left(t^{2}\right)}[\mathrm{j} \beta-\alpha / 2] \mathrm{L}_{\mathrm{PS}} \approx-1-F \cdot \frac{2}{\pi}[\mathrm{j} \beta-\alpha / 2] \mathrm{L}_{\mathrm{PS}} \approx-\exp \left([\mathrm{j} \beta-\alpha / 2] \cdot F \cdot \frac{2}{\pi} \mathrm{L}_{\mathrm{PS}}\right)
\end{aligned}
$$

where $\beta$ and $\alpha$ are the real part of the wave number (describing the phase propagation) and the attenuation coefficient, respectively. LPS is the length of the phase shifter embedded in each resonator and F is the Finesse which can be calculated as the ratio between the Free Spectral Range (FSR) and the FWHM, F=FSR / FWHM $\approx-2 \pi / \log _{n}\left(t^{2}\right)$. Equation 4 shows that the resonance results in both the phase propagation term and the attenuation being enhanced by a common multiplicative factor. As a consequence, both parameters $\mathrm{A}$ and $\Delta \varphi_{\mathrm{RF}}$ change with wavelength and reach, at the resonant wavelength $\left(\lambda_{\text {res }}\right)$, a peak enhancement factor that can be approximated as $\Gamma_{\text {peak }} \approx 2 \cdot \mathrm{F} / \pi \approx-4 / \log _{n}\left(t^{2}\right) . \Gamma_{\text {peak }}$ is a crucial figure of merit since it corresponds to the reduction of $\mathrm{V}_{\pi} \mathrm{L}$ (the product of the drive voltage needed to achieve a $\pi$ phase $\operatorname{shift}\left(\mathrm{V}_{\pi}\right)$ and the required length of the phase shifter $\left.(\mathrm{L})\right)$. As a consequence, $\Gamma_{\text {peak }}$ also corresponds to the reduction of the cumulative PIN junction length, of the modulator capacitance, and thus of the maximum achievable reduction in power consumption. Reduction of $\Gamma_{\text {peak }}$ with increasing coupling strength is illustrated in Figure 1(c). The inclusion of multiple (N) resonators loaded on each arm allows reaching the required phase shift notwithstanding a limited Finesse and enhancement factor. Thus, each RRM can exhibit a wide optical bandwidth, since it only needs to generate a fraction of the total required phase shift. In particular, given an effective refractive index variation $\pm \Delta \mathrm{n}_{\text {eff }}$ induced by the applied $\mathrm{RF}$ signal in rings loaded on either arm, $\Delta \varphi_{\mathrm{RF}}$ can be written as:

$$
\Delta \varphi_{R F}= \pm(2 \pi / \lambda) \cdot \Delta n_{e f f} \cdot \Gamma \cdot \mathrm{L}_{\mathrm{PS}} \cdot \mathrm{N}
$$

with $\Gamma$ the enhancement factor that varies with wavelength and reaches a peak at the resonance.

It is interesting to compare the proposed resonant modulator to a modulator based on straight phase shifters with an equivalent PN junction. Due to the multiplicative effect being applied to both phase shift and round-trip loss, the relevant figure of merit $\mathrm{V}_{\pi} \mathrm{L} \times \alpha$, consisting in the product of $\mathrm{V}_{\pi} \mathrm{L}$ and the linear optical losses $\alpha$ (here the total loss in each arm divided by the cumulative length of the embedded phase shifters $L=N \cdot L_{P S}$ ) remains the same. In order to reach this ideal scenario with the resonant modulator approach, it is then critical not to induce significant excess optical losses associated with the curvature of the waveguide or abrupt mode mismatch in the coupling section.

According to Equations 3 and 4, the modulation enhancement factor at a targeted optical bandwidth (constrained resonance FWHM) can be increased by reducing the resonator size (larger FSR). However, the design of a resonator combining a small radius, a significant coupling strength and a small excess loss is quite challenging. In section 3 we present the design of resonators that have been optimized for the simultaneous fulfillment of these three requirements.

\subsection{Passively biased scheme with Multimode Grating Coupler}

In the selected MZI configuration, $\Delta \varphi_{0}$ results from fabrication asymmetries between the interferometer arms. For a maximization of the modulator performance (Optical Modulation Amplitude, OMA), the MZI needs to be biased at quadrature ( $\Delta \varphi_{0}=\pi \mathrm{m}$, with $\mathrm{m}$ integer). In order to achieve this condition without the need of any active element (such as a thermal phase tuner) we propose to use a Multimode Grating Coupler (MMGC) as both a light coupling and splitting element. A MMGC is an interferometric device whose geometry is designed for equal splitting of input light into its two output waveguides. Moreover, lateral displacements of the input light source over a large range do not significantly penalize Insertion Losses (ILs) or splitting imbalance. Thus, multimode couplers have been proposed to relax the 


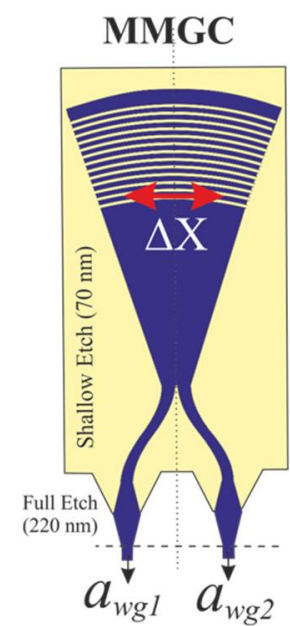

(a)

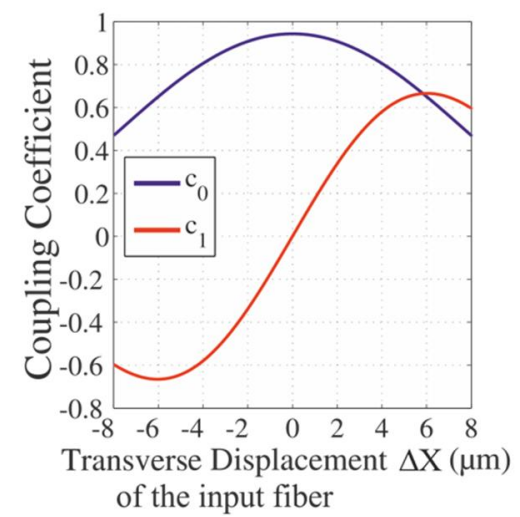

(b)

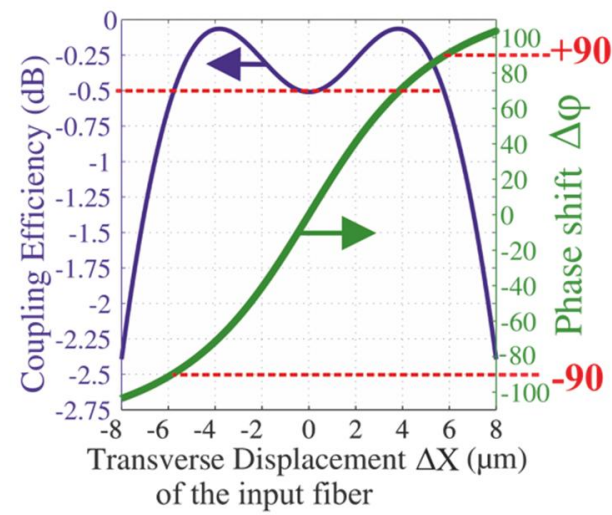

(c)

Figure 2. a) Schematic representation of a Multimode Grating Coupler (MMGC) for the injection and splitting of light in the MZI. b) Variation of the amplitude coupling coefficients into the ground and first order modes of the superstructure as a function of the transverse displacement of the input fiber $(\Delta \mathrm{X})$. c) Coupling efficiency into and relative phase shift between the two output waveguides as a function of the transverse displacement. The excess loss due to lateral displacement over the entire displacement range required to compensate for $\pm 90^{\circ}$ (providing complete compensation for fabrication asymmetries) is less than $0.5 \mathrm{~dB}$.

alignment tolerances for efficient fiber-to-chip coupling with a demonstrated three-fold improvement in comparison with conventional single mode couplers ${ }^{9,13}$. In a MMGC, the input displacement is converted into a relative phase shift between the outputs. When the MMGC is integrated in a MZI, this phase shift can be leveraged to compensate for the phase difference produced by fabrication asymmetries in the two arms.

At the input interface, a transverse displacement $(\Delta \mathrm{X})$ of the input fiber relative to the MMGC center results in varying amplitude coupling coefficients into the ground $\left(\mathrm{c}_{0}\right)$ and first order $\left(\mathrm{c}_{1}\right)$ modes of the superstructure. The MMGC is sized by design for the two modes to be in quadrature $\left(90^{\circ}\right.$ phase shifted) at the output of the device. Hence, the field amplitudes at the junction to the MZI arms ( $a_{\mathrm{wg} 1}$ and $a_{\mathrm{wg} 2}$ ) can be described according to the equations:

$$
\begin{aligned}
& a_{w g 1}(\Delta \mathrm{X})=(1 / \sqrt{2}) \cdot\left(c_{0}(\Delta \mathrm{X})+j \cdot c_{1}(\Delta \mathrm{X})\right) \\
& a_{w g 2}(\Delta \mathrm{X})=(1 / \sqrt{2}) \cdot\left(c_{0}(\Delta \mathrm{X})-j \cdot c_{1}(\Delta \mathrm{X})\right)
\end{aligned}
$$

Therefore, the phase shift $\Delta \varphi_{0}$ as a function of the lateral displacement $\Delta \mathrm{X}$ is described by:

$$
\Delta \varphi(\Delta \mathrm{X})=2 \cdot \arctan \left(-\mathrm{c}_{1}(\Delta \mathrm{X}) / \mathrm{c}_{0}(\Delta \mathrm{X})\right)
$$

The variation of the coupling coefficients $\mathrm{c}_{0}, \mathrm{c}_{1}$ as a function of the transversal displacement is illustrated in Figure 2(b) and the corresponding coupling efficiency into and phase shift in between the waveguides are plotted in Figure 2(c). In these plots, we have normalized out the loss sources also typically occurring in conventional single output Grating Couplers (GCs), namely the effect of the GC directionality for a buried oxide layer of $2 \mu \mathrm{m}$ and the suboptimal overlap with the incoming fiber mode in the in-plane direction of propagation. These additional losses amount to a total of $2.85 \mathrm{~dB}$. The input field corresponds to the mode of a standard SMF-28 fiber with a mode field diameter of $10.4 \mu \mathrm{m}$. Furthermore, the width of the MMGC (20.2 $\mu \mathrm{m}$ at the starting point of the grates) provides a ground mode field diameter of $17.2 \mu \mathrm{m}(1.65$ times larger than the input field). Fiber displacements in the range [-5.85 $\mu \mathrm{m}, 5.85 \mu \mathrm{m}]$ enable the compensation of any fabrication asymmetry in the MZI arms by means of a phase shift $\Delta \varphi_{0}$ in the range $\left[-90^{\circ}, 90^{\circ}\right]$.

The MMGC width has been set to minimize the excess loss for any displacement in the aforementioned range and assures a penalty of less than $0.5 \mathrm{~dB}$ in comparison with an optimized single-mode GC design. A thinner MMGC can reduce the insertion loss for centered alignment $(\Delta \mathrm{X}=0)$ but will introduce a higher excess loss in case the maximum compensation $(\Delta \varphi= \pm 90$ degrees) is needed. On the contrary, a wider MMGC would have the opposite effect, with a higher excess loss 
for centered alignment but a lower penalty for the largest displacements. The phase adjustment requires a precise alignment of the input fiber. The phase difference between the MMGC output waveguides is most sensitive to displacements of the fiber when the latter is positioned at the center of the MMGC. In order to adjust the phase with an error below \pm 5 degrees, the input fiber needs to be aligned with a precision better than $\pm 0.2 \mu \mathrm{m}$.

\section{RESONANTLY ENHANCED PHASE SHIFTER DESIGN}

\subsection{Resonator design with lateral PN junction}

The first device has been designed to be fabricated in the standard silicon photonics technology platform at IME A*STAR with $248 \mathrm{~nm}$ DUV optical lithography ${ }^{14}$. The optical layer stack is comprised of a buried silicon dioxide layer of $\sim 2 \mu \mathrm{m}$, a silicon waveguide core with a thickness of $220 \mathrm{~nm}$ and a $\mathrm{SiO}_{2}$ top cladding of several micrometers. The fabrication processes include a full etch step ( $220 \mathrm{~nm}$ etch depth), a shallow etch step for the definition of grating couplers and a deep etch step (130 nm etch depth) for the definition of rib waveguides with a $90 \mathrm{~nm}$ slab height. We selected the deeply etched rib waveguides for the implementation of ring resonators with embedded PN junctions. The waveguide slab allows electrical connectivity of the diode junction with the cathode metal contact at the center of the resonator and the anode contact placed at opposite sides of each ring (see Figure 3 (c)).

The resonator geometry has been specifically optimized to achieve a very compact design with high coupling strength $\left(\kappa^{2}\right.$ $\approx 0.4$ for a phase shifter enhancement factor of $\sim 8$ ), low bending loss and low excess loss in the coupling junction. The design parameters that mostly influence the bending loss are two: the radius and the resonator waveguide width. As we narrow the waveguide width and reduce the optical confinement, at tight radii below $10 \mu \mathrm{m}$ bending losses increase due to leakage through the outer slab. On the contrary, further widening of the ring's waveguide above $1 \mu \mathrm{m}$ produces negligible variations of the bending losses, because the optical mode, confined at the outer periphery of the ring, no longer grows in size and its interaction with the inner waveguide interface is no longer significant. As a result, in order to achieve low bending loss with a very compact radius, the waveguide width should be set to a value larger than $1 \mu \mathrm{m}$. However, the high modal confinement in such a wide waveguide also impedes the achievement of the required coupling strength between the bus waveguide and the cavity. Here, an excessive reduction of the coupling gap is not a viable option because it typically results in significant excess loss at the coupling junction and a device that is more sensitive to fabrication variations.

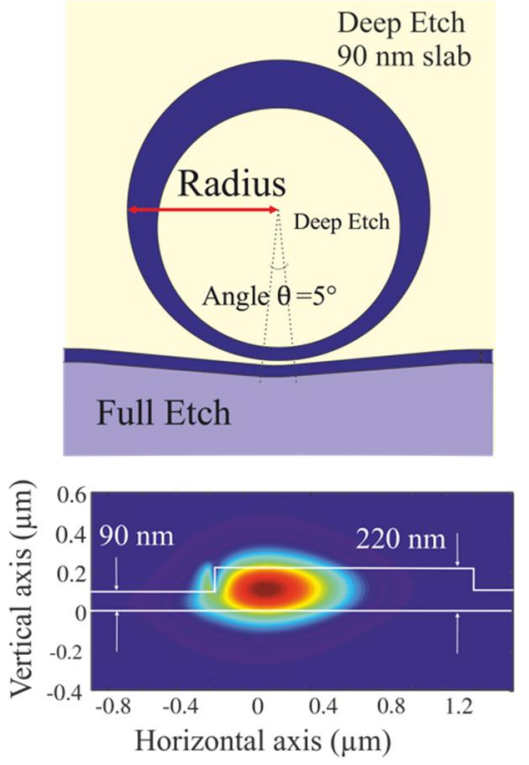

(a)
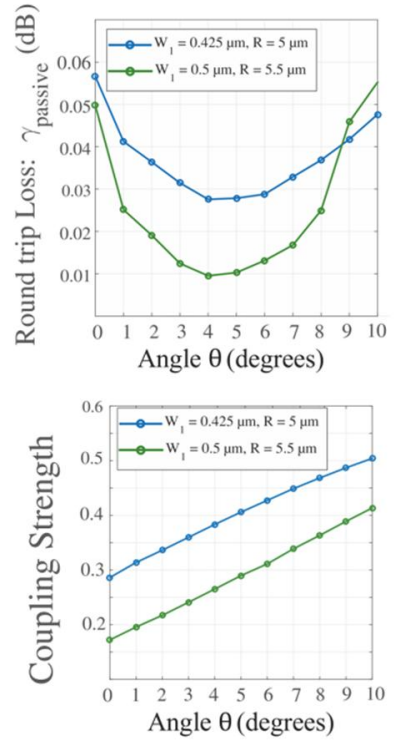

(b)
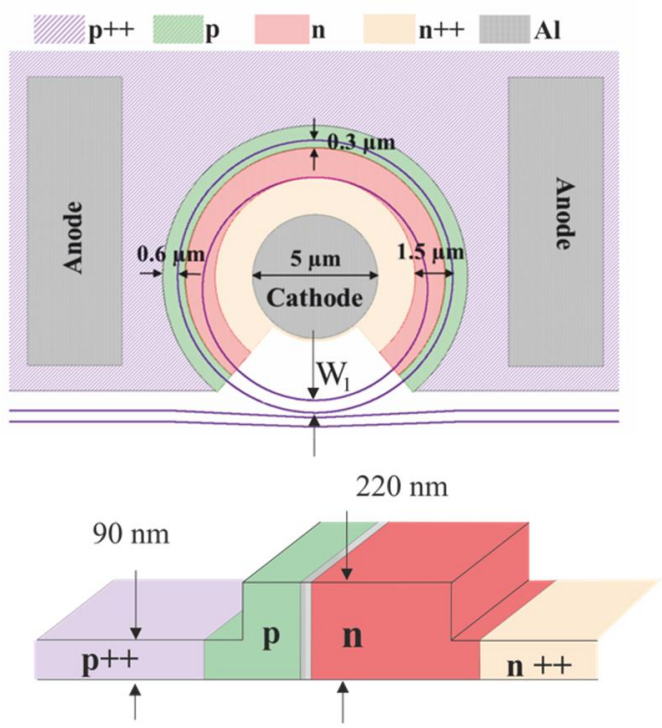

(c)

Figure 3. (a) Schematic layout of the proposed resonator with lateral PN junction (top) and bend mode field (transverse electric field component) for a cavity radius of $5 \mu \mathrm{m}$ and a width of $1 \mu \mathrm{m}$ (bottom). (b) Simulation results: Passive round trip loss (top) and coupling coefficient $\left(\kappa^{2}\right)$ as a function of the coupling section angle $\theta$ and different resonator configurations. (c) Layout with implanted regions and electrical contacts (top) and cross section of the implanted ring waveguide (bottom). 
In order to overcome these problems, we proposed a novel ring resonator geometry with three key features. First, the ring waveguide width adiabatically transitions from a wide cross section $(1.58 \mu \mathrm{m})$ to a narrow width in the coupling region with marginal excitation of high-order modes. The narrow width expands the evanescent field of the cavity mode and thus increases the coupling strength to and from the bus waveguide. On the other hand, the wider waveguide section reduces the round-trip bending loss. Second, the radiative losses that would otherwise occur in the narrow waveguide region are suppressed by fully etching the slab on the opposite side of the bus waveguide. In this manner, the asymmetrical profile of the bus waveguide provides a high-index contrast interface that prevents the leakage through the slab. Finally, the high coupling strength and the smooth modal conversion in the coupling section are facilitated by tapering the gap ${ }^{15}$ with an optimized bus waveguide bend angle $\theta$ (see Figure 3 (a) and (b)). Importantly, the minimum gap between the bus waveguide and the resonator is set to $210 \mathrm{~nm}$ in order to guarantee properly resolved fabrication.

We have optimized the different design parameters by means of 3D-FDTD simulations. We first set the ring width in the wider section to a value $>1 \mu \mathrm{m}$ and we modified both the ring width in the narrow section $(0.425 \mu \mathrm{m})$ and the bus waveguide width $(0.37 \mu \mathrm{m})$ for a considerable coupling strength (enabled by favorable phase matching). Then, we varied the coupling section angle $\theta$ until the round-trip loss reached a minimum, assisting in the adiabatic transition between the supermodes in the coupler and the cavity mode in the wider section. Finally, the targeted coupling strength $\left(\kappa^{2} \approx 0.4\right)$ was obtained by increasing the ring width in the narrow section. With a $5 \mu \mathrm{m}$ resonator radius, the round-trip "passive" loss (i.e., in the absence of implants) per resonator ( $\gamma_{\text {passive }}$ without including propagation losses due to absorption or side-wall roughness) reaches a minimum of $\sim 0.03 \mathrm{~dB}$ for a coupling section angle of $\theta \approx 5^{\circ}$. For smaller angle values, the roundtrip loss is penalized by an abrupt mode conversion in the coupling region. On the other hand, larger angle values lead to a stronger coupling from the bus waveguide mode into higher order modes of the cavity. These higher order modes are quickly radiated out due to the compact resonator radius, penalizing the round-trip loss. The value of $\gamma_{\text {passive }}$ can be further reduced to below $0.01 \mathrm{~dB}$ by increasing the ring radius above $5.5 \mu \mathrm{m}$.

For the implementation of the embedded PN diode we selected doping concentrations with average values of $1.5 \mathrm{e}-18 \mathrm{~cm}^{-}$

${ }^{3}$ for both the $\mathrm{p}$ - and $\mathrm{n}$-doped regions. These concentrations were specifically optimized for high-speed single RRMs aimed at amplitude modulation ${ }^{16}$, leading to a relatively high efficiency in reverse bias for a straight phase shifter ( $\mathrm{V} \pi \cdot \mathrm{L}$ of 1.3 $\mathrm{V} \cdot \mathrm{cm})$ but also high propagation loss due to carrier absorption $(\sim 5.5 \mathrm{~dB} / \mathrm{mm})$. The depletion region was placed near the outer edge of the resonator $(0.3 \mu \mathrm{m})$ in order to get a maximized overlap with the optical field. When compared to a straight phase shifter with optimized width (reference design with $400 \mathrm{~nm}$ width) the locally wider cross section of the resonator waveguide width introduces a cumulative $15 \%$ penalty due to the enlarged mode profile. As a result, the effective phase shift enhancement factor (when compared to the straight phase shifter) is reduced from 8 down to 6.8. Finally, we selected the position of the highly doped regions based on modal analysis, avoiding a high absorption due to interaction of the carriers with the resonator mode (additional propagation loss of less than $1 \mathrm{~dB} / \mathrm{cm}$ ) but also providing a reduced diode series resistance. The surrounding $\mathrm{p}++$ contact region was spaced $0.6 \mu \mathrm{m}$ from the outer resonator edge. The inner $\mathrm{n}++$ region was bounded by a circle spaced $1.5 \mu \mathrm{m}$ from the outer sidewall of the cavity. Furthermore, a cylindrical aluminum via with a $5 \mu \mathrm{m}$ diameter was placed at the center of the resonator for the interconnection with the electrode. Figure 3(c) depicts the schematic layout with the different implant regions.

The $\mathrm{p}$ - and $\mathrm{n}$-doped regions exclude the coupling region to avoid optical loss in the bus waveguide, surrounding $3 / 4$ of the resonator and extending an unfolded phase shifter length of $22 \mu \mathrm{m}$ for a ring radius of $5 \mu \mathrm{m}(4.7 \mu \mathrm{m}$ radius for the PN junction). The single resonator phase shifter exhibits a capacitance of $14.4 \mathrm{fF}$ and a series resistance of $240 \Omega$. The $-3 \mathrm{dBe}$ intrinsic cut-off frequency of the phase shifter is therefore $f_{R C}=45.7 \mathrm{GHz}$.

\subsection{Resonator design with vertical PIN junction}

The modulation efficiency of the presented resonantly-enhanced phase shifter can be further enhanced with several design improvements. First, a resonator waveguide cross section with a thicker waveguide core and a thinner slab thickness allows a further reduction of the resonator size. According to Equations 3 and 4, for a targeted optical bandwidth (FWHM), a more compact resonator (smaller R) leads to a larger FSR and Finesse and therefore a larger modulation enhancement. Furthermore, a vertical junction enables a higher overlap with the optical field, avoiding the penalization due to the enlarged bend mode profile that occurs with lateral junctions.

The second device has been designed to be fabricated in a customized technology platform with a silicon waveguide core thickness of $290 \mathrm{~nm}$. The layer stack is created by successive steps of silicon epitaxial overgrowth and selective ion implantation assisted by optical lithography ${ }^{17,18}$. These processes allow the definition of a vertical PIN junction with the upper implanted layer (n-type silicon doped with Phosphorous) extending just a few tens of nanometers up to a height of 


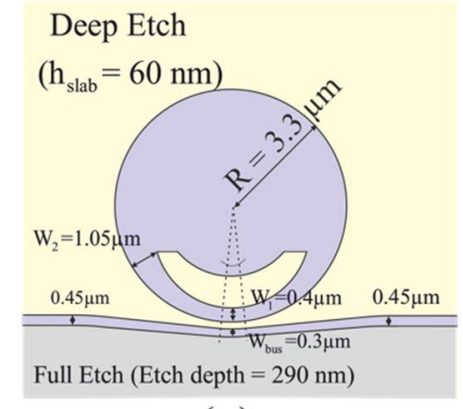

(a)

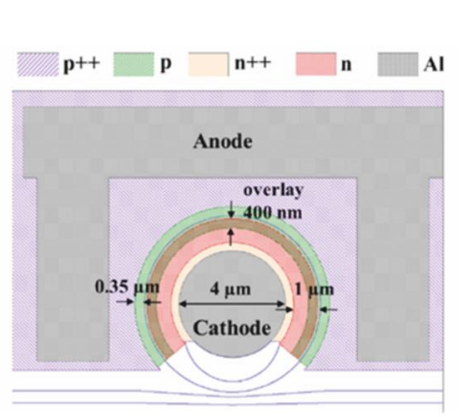

(d)

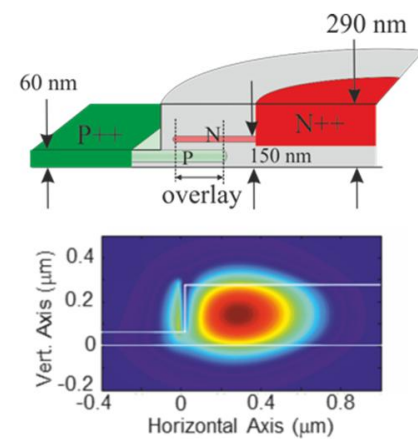

(b)

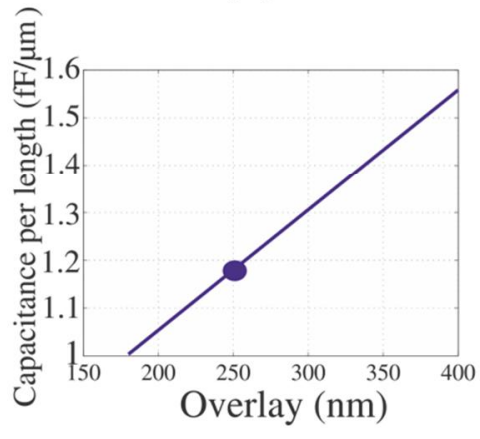

(e)

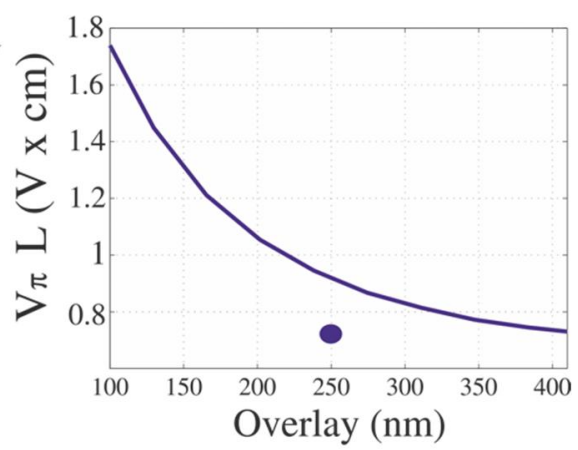

(c)

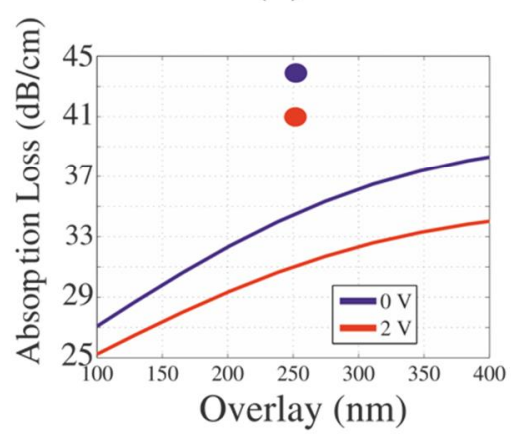

(f)

Figure 4. (a) Schematic layout of the proposed resonator with vertical PIN junction. (b) Cross section of the implanted resonator waveguide (top) and bend mode field (transverse electric field component) for a radius of $3.3 \mu \mathrm{m}$ (bottom). (c) Modulation efficiency $\left(\mathrm{V}_{\pi} \mathrm{L}\right)$ as a function of the overlay between $\mathrm{p}$ - and n-doped regions. (d) Layout of the proposed resonator with electrical contacts. Junction capacitance (e) and absorption loss (f) both as a function of the overlay. Metrics for a reference phase shifter with straight waveguide (150 nm slab) are marked with points.

$150 \mathrm{~nm}$ (see Figure 4(b)). In a straight waveguide phase shifter design, a symmetric etch depth for rib waveguide definition must not exceed $140 \mathrm{~nm}(150 \mathrm{~nm}$ slab) in order to avoid cutting the connection to the cathode through the n-type layer. This constraint does not apply to the case of a microdisk, since the etch is only applied to one side of the junction. The external sidewall of our resonator is defined by etching down to the limit given by the bottom p-type Boron implanted layer, leaving a lateral slab of $60 \mathrm{~nm}(230 \mathrm{~nm}$ etch depth) for the electrical connection with the surrounding anode (see Figure 4(b)). On the other side, the silicon layer in the inner part of the resonator is not etched for connection to the central cathode contact.

Moreover, a deeply etched region in the coupling section of the resonator enables the local definition of a ring waveguide that can be easily phase matched to a thin bus waveguide. Similarly to the design with lateral junction, the required coupling strength is facilitated by an asymmetric bus waveguide (fully etching the outer slab) that enhances the evanescent field towards the cavity mode. Here again, by tapering the ring waveguide width in the coupling section we achieve an adiabatic conversion of the resonator mode profile without the excitation of high order modes. For a ring waveguide width larger than $1.05 \mu \mathrm{m}$, the interaction of the optical field with the inner waveguide sidewall is negligible and the deep etch region can be stopped without significant penalization in loss due to mode conversion. Outside the coupling section, the resonator continues in a microdisk configuration with electrical connection to the central cathode. Figure 4(d) depicts a top view schematic of the proposed device.

Based on modal analysis simulations of the circulating cavity mode, we set the resonator radius to $3.3 \mu \mathrm{m}$ for negligible radiative losses. Then, based on an iterative design process by means of 3D-FDTD simulations we chose a minimum resonator waveguide width in the coupling section of $0.4 \mu \mathrm{m}$, a bus waveguide width of $0.3 \mu \mathrm{m}$ and a gap of $180 \mathrm{~nm}$. The coupling angle $\theta$ was also set to 5 degrees in a trade-off between coupling losses $\left(\gamma_{\text {passive }}=0.014 \mathrm{~dB}\right)$ and coupling strength $\left(\kappa^{2} \approx 0.31\right)$. The compact resonator size allows increasing the enhancement factor to 10.8 without further penalizing the optical bandwidth. 
Details about the different fabrication steps for the definition of the vertical PIN junction and the carrier concentrations can be found in ref. 18. The bottom p-type region reaches a peak carrier concentration of $5.3 \mathrm{e}^{18 \mathrm{~cm}^{-3}}$ at a height of 35.8 $\mathrm{nm}$ above the Buried Oxide (BOX) - Silicon interface, whereas the n-type region is limited to a region of less than $25 \mathrm{~nm}$

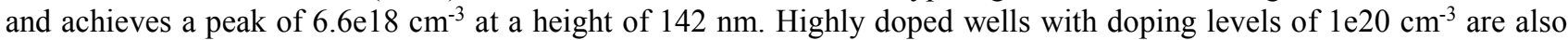
defined on the inner and outer parts of the resonator in order to provide electrical contact to the aluminum interconnects. For an excess loss of less than $1 \mathrm{~dB} / \mathrm{cm}$, we kept a distance of $1 \mu \mathrm{m}$ to the inner $\mathrm{n}++$ region and $0.35 \mu \mathrm{m}$ to the outer $\mathrm{p}++$ region, both measured from the external sidewall of the resonator. At the center of the resonator there is space for a cylindrical aluminum via with a diameter of $4 \mu \mathrm{m}$. The coupling section is not implanted in order to avoid the absorption losses in the bus waveguide. The layout with the different implantation layers of the resonator and the aluminum vias is depicted in Figure 4(d).

The lithographic definition of the implanted regions allows freely choosing the overlay between the n-type and p-type layers. To set this parameter we simulated the bend mode with FIMMWAVE after importing the 2D carrier distribution profiles (simulated with COMSOL) for different applied voltages. As we can see in Figure 4(c), it is possible to enhance the modulation efficiency (calculated from $0 \mathrm{~V}$ to $2 \mathrm{~V}$ reverse bias) by extending the overlay between the $\mathrm{n}$-type and $\mathrm{p}$ type regions. However, a larger overlay increases both the optical absorption and the capacitance per length. For comparison purposes, we represent with dots the values obtained for the optimized straight phase shifter with a waveguide width of $470 \mathrm{~nm}$, a slab height of $150 \mathrm{~nm}$, and an overlay of $250 \mathrm{~nm}$. Although, for the same $250 \mathrm{~nm}$ overlay, the resonant phase shifter configuration exhibits a lower modulation efficiency $\left(\mathrm{V}_{\pi} \mathrm{L}=0.95 \mathrm{~V} \cdot \mathrm{cm}\right.$ prior to applying the resonant enhancement) than the straight phase shifter $\left(\mathrm{V}_{\pi} \mathrm{L}=0.74 \mathrm{~V} \cdot \mathrm{cm}\right)$, the absorption loss is also significantly smaller $(3.4 \mathrm{~dB} / \mathrm{mm}$ instead of $4.4 \mathrm{~dB} / \mathrm{mm}$ for $0 \mathrm{~V}$ ). This reduction in absorption loss is due to a smaller overlap with the p-doped layer as a result of the deeper etching. In order to recover a modulation efficiency of $0.74 \mathrm{~V} \cdot \mathrm{cm}$, we selected an overlay of $400 \mathrm{~nm}$, which still provides a significant improvement in absorption loss $(3.83 \mathrm{~dB} / \mathrm{mm}$ for $0 \mathrm{~V})$. For this overly value, the capacitance per length increases to $1.55 \mathrm{fF} / \mu \mathrm{m}$ (calculated for $0 \mathrm{~V}$ ) as compared to $1.17 \mathrm{fF} / \mu \mathrm{m}$ for the straight phase shifter reference design.

In the resonator design with vertical junction, the phase shifter extends an unfolded cavity length of $13.6 \mu \mathrm{m}$. We calculated a total round trip loss of $\gamma=0.061 \mathrm{~dB}$, including the round-trip loss due to carrier absorption $(0.052 \mathrm{~dB})$ and $\gamma_{\text {passive. }}$. According to the enhancement factor of this compact design, the optical excess loss introduced by the cavity at the resonant wavelength increases to $0.66 \mathrm{~dB}$. The calculated total diode resistance per resonator including the highly doped regions is $296 \Omega$. Moreover, the total capacitance calculated for $0 \mathrm{~V}$ is $21.2 \mathrm{fF}$. Therefore, the $-3 \mathrm{dBe}$ intrinsic cut-off frequency of the phase shifter is $f_{R C}=25.3 \mathrm{GHz}$.

Table 1 summarizes the performance and figures of merits of the two resonator designs.

Table 1. Characteristics and figures of merits of the two resonantly enhanced phase shifter designs with lateral and vertical PIN junctions.

\begin{tabular}{|c|c|c|c|c|c|c|c|c|c|}
\hline & \multicolumn{3}{|c|}{$\begin{array}{l}\text { Phase shifter performance without } \\
\text { resonant enhancement }\end{array}$} & \multirow{2}{*}{$\begin{array}{c}\text { Resonance } \\
\text { FWHM } \\
\text { (nm) }\end{array}$} & \multirow{2}{*}{$\begin{array}{c}\text { Peak } \\
\text { Enhancement } \\
\text { Factor at } \lambda_{\text {res }}\end{array}$} & \multirow{2}{*}{$\begin{array}{l}\text { Res. Radius }(\mu \mathrm{m}) / \\
\text { Phase Shifter } \\
\text { Length Lps }(\mu \mathrm{m})\end{array}$} & \multicolumn{2}{|c|}{$\begin{array}{c}\text { Performance at } \\
\lambda_{\text {res }}\end{array}$} & \multirow{2}{*}{$\begin{array}{c}\text { Intrinsic } \\
\text { Electrical } \\
\text { Cut-off Freq. } \\
f_{\mathrm{RC}}(\mathrm{GHz})\end{array}$} \\
\hline & $\begin{array}{c}\mathrm{V}_{\pi} \cdot \mathrm{L} \\
(\mathrm{V} \cdot \mathrm{cm})\end{array}$ & $\begin{array}{c}\alpha^{(1)} \\
(\mathrm{dB} / \mathrm{cm})\end{array}$ & $\begin{array}{c}\mathrm{V}_{\pi} \cdot \mathrm{L} \times \alpha^{(1)} \\
(\mathrm{V} \cdot \mathrm{dB})\end{array}$ & & & & $\begin{array}{l}\mathrm{IL}^{(2)} \\
(\mathrm{dB})\end{array}$ & $\begin{array}{c}\Delta \varphi_{\mathrm{RF}}{ }^{(3)} \\
\text { (degrees) }\end{array}$ & \\
\hline $\begin{array}{l}\text { Lateral Junction } \\
\text { Resonator }\end{array}$ & 1.3 & 55 & 71.5 & 2 & 8 & $5 / 22$ & 1.2 & 3.8 & 45.7 \\
\hline $\begin{array}{l}\text { Vertical Junction } \\
\text { Resonator }\end{array}$ & 0.74 & 36 & 26.6 & 2.2 & 10.8 & $3.3 / 13$ & 0.66 & 7.2 & 25.3 \\
\hline
\end{tabular}

(1) Without including excess loss in the coupling section.

(2) Including excess loss in the coupling section.

(3) Calculated from $0-2 \mathrm{~V}$.

\section{MZM DESIGN WITH RESONATOR ARRAYS}

\subsection{Modulator Designs}

For selecting the number of resonators per array $(\mathrm{N})$, we maximized the Optical Modulation Amplitude (OMA) of the MZM with $2 \mathrm{~V}_{\mathrm{pp}}$ signaling in push-pull driving. For each design, we obtained the OMA by inserting Eq. (5) into either Eq. (1) or (2) and by calculating the difference between the maximum output power $\mathrm{P}_{1}$ (high level) and the minimum output power $\mathrm{P}_{0}$ (low level) for the given applied voltage swing, and normalizing both to the input power $\left(\mathrm{P}_{\text {in }}\right)$, following the definition: 
RE-MZM with lateral PIN junction resonators

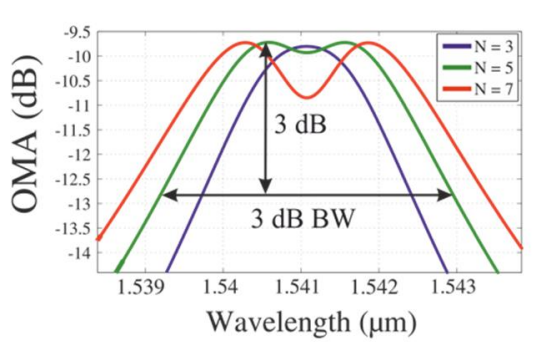

(a)

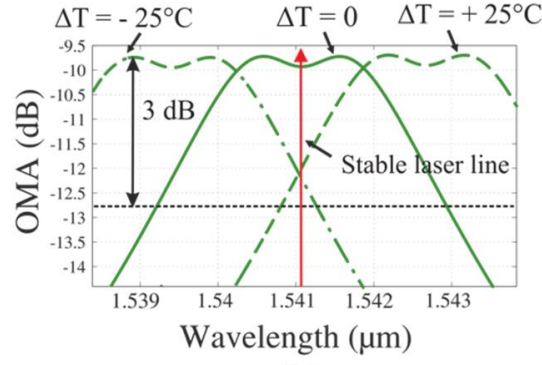

(b)
RE-MZM with vertical PIN junction resonators

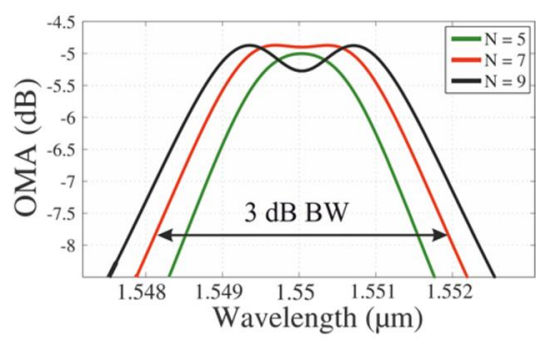

(c)

Figure 5. (a) Calculated OMA of the RE-MZM with lateral junction resonators and $2 \mathrm{~V}_{\mathrm{pp}}$ push-pull driving as a function of wavelength for $\mathrm{N}=3$ (blue), 5 (green), and 7 (red) resonators loaded on each arm. (b) OMA spectra for a RE-MZM with $\mathrm{N}=5$ and operating temperatures shifted by $\Delta \mathrm{T}=-25^{\circ} \mathrm{C}$ (dashed-dot), $0^{\circ} \mathrm{C}$ (solid), and $+25^{\circ} \mathrm{C}$ (dashed). (c) Calculated OMA of the RE-MZM with vertical junction resonators and $2 \mathrm{~V}_{\mathrm{pp}}$ push-pull driving as a function of wavelength for $\mathrm{N}=5$ (green), 7 (red), and 9 (black) resonators loaded on each arm.

$$
\text { OMA }[\mathrm{dB}]=10 \cdot \log _{10}\left(\left(\mathrm{P}_{1}-\mathrm{P}_{0}\right) / \mathrm{P}_{\text {in }}\right)
$$

where we assumed that all cavities are exactly identical and that $\Delta \varphi_{0}$ can be canceled throughout the entire optical bandwidth. This metric combines the modulation penalty due to the finite drive voltage and the actual insertion losses. According to Eq. (5), a higher $\mathrm{N}$ leads to a larger accumulated phase shift. However, it also increases the total insertion losses introduced by the RRM arrays. Figure 5(a) shows the calculated OMA for RE-MZMs with lateral PN junction and with $\mathrm{N}=3,5$, and 7 under a $2 \mathrm{~V}_{\mathrm{pp}}$ differential drive voltage in push-pull configuration. Increasing $\mathrm{N}$ from 3 to 7 reduces the OMA at the resonant wavelength as a consequence of peak attenuation. Since moderately detuning the optical carrier from the resonance wavelength results in the Insertion Losses (ILs) being reduced faster than the phase shift efficiency, two side peaks appear in the wavelength-dependent OMA. As a beneficial side effect, a higher $\mathrm{N}$ also extends the optical bandwidth of the device by further splitting the positions of the peaks. The peak OMA value stops increasing for a sufficiently large $\mathrm{N}(\mathrm{N}>3$ for the phase shifter design with lateral PN junction) due to the nonlinearity in the transfer function of the MZI with the applied phase shift (Eq. (1)). A higher $\mathrm{N}$ also implies a larger modulator capacitance and a smaller resistance, which in turns leads to the requirement of a smaller driver output resistance to maintain a high electrical bandwidth close to the cutoff frequency of the diode ${ }^{19}$. The optical Bandwidth (BW) has been defined as the wavelength range where the OMA is penalized by less than $3 \mathrm{~dB}$ relative to its peak.

For the device with resonator arrays with embedded lateral PN junctions, we selected five resonators on each arm $(\mathrm{N}=5)$, resulting in a good compromise between insertion loss, capacitance, and optical bandwidth. Consequently, the PN junction extends over a cumulative length of $110 \mu \mathrm{m}$ on each arm. Moreover, each arm exhibits a total capacitance of $72 \mathrm{fF}$ and a series resistance of $48 \Omega$, leading to an intrinsic electrical cutoff frequency of $46 \mathrm{GHz}$ and a total RF power consumption of $\sim 5 \mathrm{~mW}$ dissipated in the two arms at $32 \mathrm{Gbps}$ and $2 \mathrm{~V}_{\mathrm{pp}}$. As illustrated in Fig. 3(b), variations in the operating temperature of the modulator produce a collective shifting of the resonances of about $72 \mathrm{pm} /{ }^{\circ} \mathrm{C}$. As a result, the modulation of a stable laser emitting at $\lambda_{\text {res }}$ is expected to tolerate a thermal drift of $\pm 27^{\circ} \mathrm{C}\left(54^{\circ} \mathrm{C}\right.$ temperature range $)$.

Table 2. Performance comparison between the two RE-MZM designs proposed in this work

\begin{tabular}{|c|c|c|c|c|c|c|c|c|c|}
\hline & $\begin{array}{c}\text { Number of } \\
\text { Resonators } \\
\text { per Array } \\
\text { (N) }\end{array}$ & $\begin{array}{l}\text { Cumulative } \\
\text { Phase Shifter } \\
\text { Length per } \\
\text { Arm }(\mu \mathrm{m})\end{array}$ & $\begin{array}{c}\text { IL at } \\
\lambda_{\text {res }} \\
(\mathrm{dB})^{(1)}\end{array}$ & $\begin{array}{c}\text { ER at } \\
\lambda_{\text {res }} \\
(\mathrm{dB})^{(1)}\end{array}$ & $\begin{array}{l}\text { OMA } \\
(\mathrm{dB})^{(1)}\end{array}$ & $\begin{array}{c}3 \mathrm{~dB} \text { Optical } \\
\text { Bandwidth } \\
(\mathrm{nm}) / \text { Temp. } \\
\text { Range }\left({ }^{\circ} \mathrm{C}\right)\end{array}$ & $\begin{array}{l}\text { Capacitance } \\
\text { per arm } \\
\text { (fF) }\end{array}$ & $\begin{array}{l}\text { Electrical } \\
\text { cutoff } \\
\text { frequency } \\
(\mathrm{GHz})^{(2)}\end{array}$ & $\begin{array}{c}\text { RF Energy } \\
\text { Consumption } \\
\text { (fJ/bit) }\end{array}$ \\
\hline $\begin{array}{l}\text { RE-MZM with } \\
\text { Lateral Junction } \\
\text { Resonator Arrays }\end{array}$ & 5 & 110 & 5.7 & 3 & -9.7 & $3.9 / 54$ & 72 & 42.5 & 144 \\
\hline $\begin{array}{l}\text { RE-MZM with } \\
\text { Vertical Junction } \\
\text { Resonators Arrays }\end{array}$ & 7 & 95.3 & 4.6 & 8.8 & -5.6 & $3.9 / 54$ & 148 & 23 & 296 \\
\hline
\end{tabular}

(1) Peak value calculated for an RF signal with $2 \mathrm{~V}_{\mathrm{pp}}$ and push-pull driving.

(2) Electrical cut-off frequency considering a driver with $4 \Omega$ output impedance. 
For the modulator with vertical junction resonators, we selected seven resonators on each arm $(\mathrm{N}=7)$ to also reach a temperature operating range larger than $50^{\circ} \mathrm{C}$ by means of a $3 \mathrm{~dB}$ optical bandwidth larger than $3.6 \mathrm{~nm}$. In this design the maximum insertion loss is limited to $4.6 \mathrm{~dB}$, the OMA achieves a peak value of $-5.6 \mathrm{~dB}$ and the vertical junction extends a cumulative length of $95 \mu \mathrm{m}$ on each arm. On the other hand, this modulator exhibits a larger capacitance (148 fF per arm) than the device with lateral junction, but the electrical cut-off frequency still exceeds $20 \mathrm{GHz}$ with the selected driver.

Table 2 summarizes different metrics for the two designed modulators, such as the total peak Insertion Loss (IL) of the RRM arrays at $\lambda_{\text {res, }}$, the cumulative length of the unfolded phase shifter $\left(N \cdot L_{P S}\right)$, the Extinction Ratio (ER) and OMA featured by the modulator at $2 \mathrm{~V}_{\mathrm{pp}}$ differential driving, the optical bandwidth / temperature range of operation corresponding to a $3 \mathrm{~dB}$ penalization of the OMA, the capacitance per arm $(\mathrm{C})$, as well as the required RF energy per bit ${ }^{19}$ $\mathrm{E}_{\mathrm{bit}}=\mathrm{C} \times \mathrm{V}^{2} / 2$.

\subsection{Experimental results}

Figure 6(a) shows a microscope image of the RE-MZM modulator with lateral PN junction fabricated at IME A*STAR. The electro-optical characterization of the modulator in a $50 \Omega$ environment revealed a cutoff frequency of $23.5 \mathrm{GHz}$ (figure 6(b)) mainly limited by the RC time constant as penalized by the $50 \Omega$ driver output impedance. As depicted in Figure 6(c), we determined an optical insertion loss of $5.7 \mathrm{~dB}$ at the resonant wavelength $\lambda_{\text {res }}=1542.9 \mathrm{~nm}$, consistent with our modeling results. This insertion loss level is slightly above that of the reference modulator $(\mathrm{IL}=4.5 \mathrm{~dB})$ with straight phase shifters (waveguide width of $400 \mathrm{~nm}$ for optimized overlap) fabricated in the same wafer. The difference is due to $0.24 \mathrm{~dB}$ excess loss per resonator associated with waveguide junction losses and bending losses not present in the reference case. This corresponds to a multiplicative factor of $8.5 \times$ for the losses associated with the unfolded $110 \mu \mathrm{m}$ junction length, i.e., the multiplicative factor is slightly above the resonator $\Gamma_{\text {peak }}=8$ due to the excess loss in bending and coupling sections.

Moreover, after biasing the interferometer to its quadrature point at $\lambda_{\text {res, }}$ we applied DC reverse-biased drive voltages of 2 $\mathrm{V}_{\mathrm{pp}}$ and $4 \mathrm{~V}_{\mathrm{pp}}$ in push-pull configuration and measured peak extinction ratios of $3 \mathrm{~dB}$ and $6 \mathrm{~dB}$, respectively (Figure 6(c)). These correspond to penalizations of the OMA by, respectively, $-4.8 \mathrm{~dB}$ and $-2.2 \mathrm{~dB}$ due to finite drive voltages.

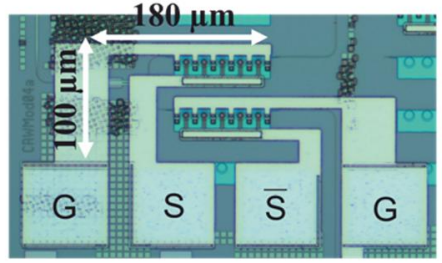

(a)

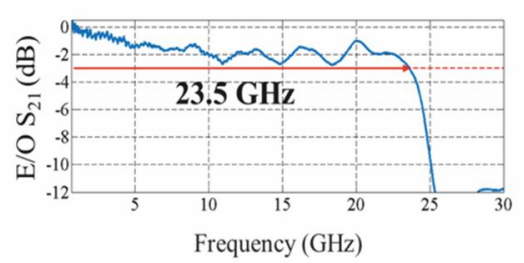

(b)

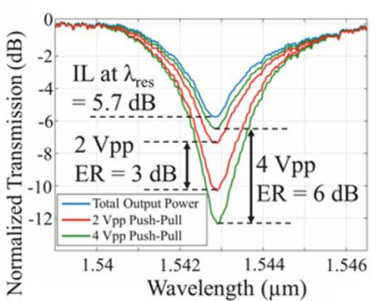

(c)

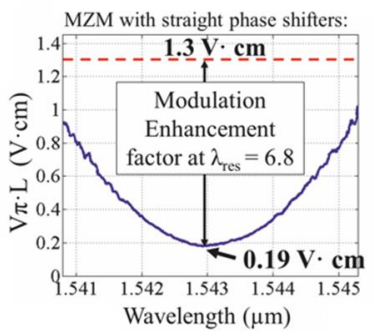

(d)

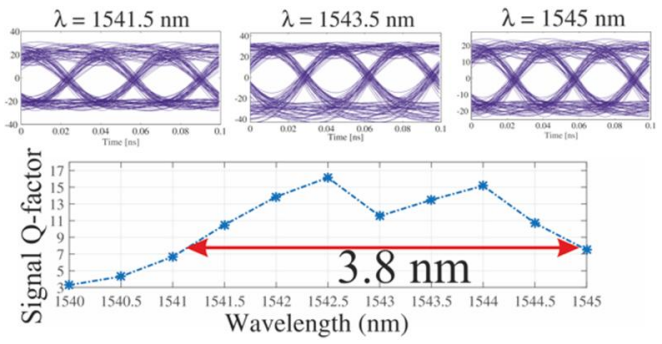

(e)

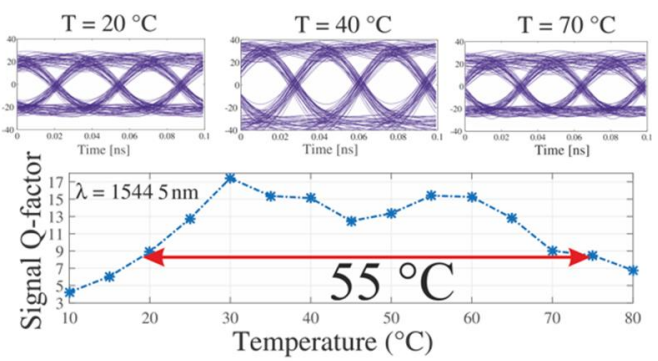

(f)

Figure 6. a) Micrograph of the fabricated RE-MZM with lateral PN junction resonators. b) Electro-optical transmission of the fabricated modulator measured in a $50 \Omega$ environment. c) DC optical transmission measurements for reverse-biased drive voltages of $2 V_{p p}$ and $4 V_{p p}$. d) RE-MZM efficiency as a function of wavelength. The red dashed line indicates the efficiency of the conventional straight phase shifter with equivalent PN lateral junction. e) Eye diagrams and extracted signal Q-factors as a function of wavelength at a fixed temperature of $25^{\circ} \mathrm{C}$ and $\mathrm{f}$ ) as a function of temperature for a fixed wavelength of $1544.5 \mathrm{~nm}$. 
Considering the cumulative RE-MZM PN junction length $(110 \mu \mathrm{m})$ and its DC response, we extract an enhanced modulation efficiency $\mathrm{V}_{\pi} \cdot \mathrm{L}$ of $0.19 \mathrm{~V} \cdot \mathrm{cm}$ at $\lambda_{\text {res, }}$, which represents a reduction by a factor of 6.8 relative to the reference case with straight phase shifters $(1.3 \mathrm{~V} \cdot \mathrm{cm})$.

We also evaluated the modulator's wavelength and temperature ranges of operation by recording eye diagrams at $30 \mathrm{Gbps}$ and extracting the corresponding signal Q-factors at different wavelengths (Figure 6(e)) and different temperatures (Figure 6(f)). At a wavelength of $1544.5 \mathrm{~nm}$ (with a signal Q-factor that exceeds the value at $\lambda_{\text {res }}$ due to reduced insertion losses), the signal Q-factor remained within a factor 2 of its peak value within a $55^{\circ} \mathrm{C}$ temperature range (from $20^{\circ} \mathrm{C}$ to $75^{\circ} \mathrm{C}$ ). At a fixed temperature of $25^{\circ} \mathrm{C}$, the signal Q-factor also remained within a factor of 2 of its peak value within a $3.8 \mathrm{~nm}$ wavelength range (from 1541.1 to $1544.9 \mathrm{~nm}$ ).

In the fabricated modulator, the net effect of both increased insertion losses and enhanced modulation efficiency is just a $25 \%$ increase in the figure of merit $\mathrm{V}_{\pi} \cdot \mathrm{L} \times \alpha$ due to bending and junction losses. We estimate the reduction in RF energy consumption ( $\mathrm{E}_{\mathrm{bit}}$ ) compared to a reference MZM driven as a series of lumped elements with a distributed driver. If the linear modulator is sized to achieve the same extinction ratio at a given drive voltage, the RE-MZM power consumption is reduced by a factor of 6.8 due to the smaller capacitance. However, since the RE-MZM exhibits $1.2 \mathrm{~dB}$ additional insertion losses, it needs to be operated with a $32 \%$ larger drive voltage in order to obtain the same OMA as the reference modulator, which reduces the effective power enhancement to a factor of 4.

A similar analysis can be done for the design with vertical PIN junction that exhibits a resonance enhancement factor of 10.8. When compared to an optimized modulator with straight phase shifters (rib slab height of $150 \mathrm{~nm}^{18}$ ) and sized to achieve the same extinction ratio, the RE-MZM solution allows a reduction of 7.7 in the power consumption. In this case, the insertion loss of the RE-MZM is penalized by only $0.1 \mathrm{~dB}$ relative to the linear modulator but the straight solution exhibits a $25 \%$ smaller capacitance.

Importantly, the reduction in modulator length for both designs removes the necessity of a distributed driver or a traveling wave (TW) configuration. The power consumption in a TW MZM would be $\sim 5 \times$ higher than in a well-designed

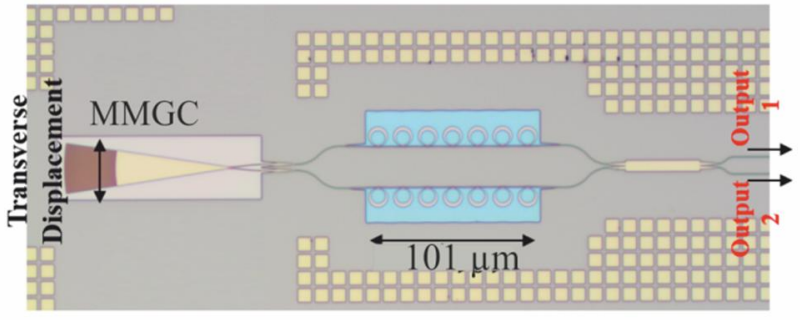

(a)

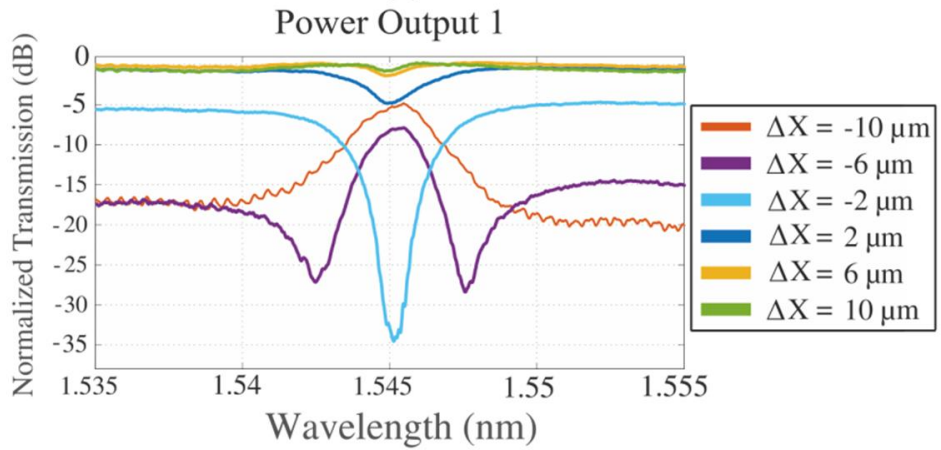

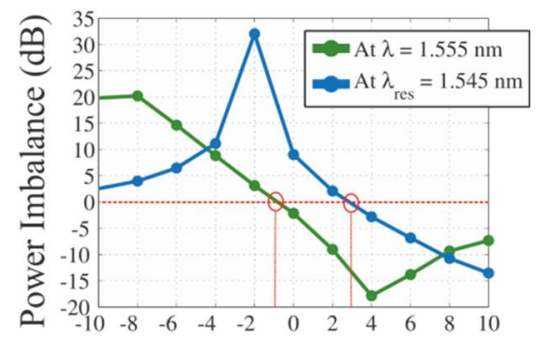

Transverse Displacement $\Delta \mathrm{X}(\mu \mathrm{m})$

(c)

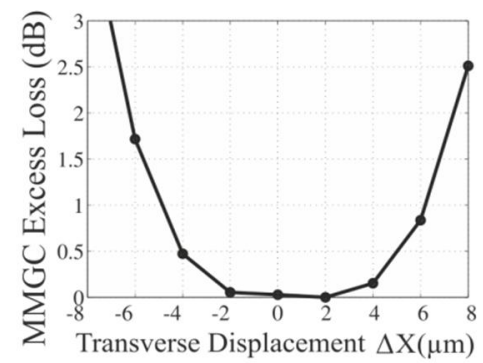

(d)

(b)

Figure 7. a) Microscope picture of the fabricated passively balanced MZI with MMGC as at the input and arrays of resonators on each arm. b) Measured power in output port 1, normalized to the maximum transmission for different values of transverse displacement of the input fiber. c) Power imbalance as a function of the transverse displacement of the input fiber measured at the resonant wavelength $\left(\lambda_{\text {res }}=1545 \mathrm{~nm}\right)$ and at a wavelength far from the resonance $\left(\lambda_{\text {res }}=1555 \mathrm{~nm}\right)$. The fiber positions for low imbalance (quadrature condition with $\Delta \varphi_{0}=0$ ) are marked with red circles. d) Excess loss introduced by the transverse displacement of the input fiber (calculated by summing the power levels measured on both output ports). 
lumped element configuration ${ }^{18}$. Compared to the TW case or factoring in the power overhead dissipated inside a distributed driver, the power enhancement factor would be significantly higher $(\sim 20 \times$ and $\sim 38 \times$, respectively for the REMZMs with lateral and vertical junctions).

Finally, we also characterized MZI test structures with a MMGC at the input and arrays of cascaded resonators on each arm. These passive structures reproduce the resonantly enhanced MZM configuration presented in the previous section (MZM with lateral junction). For these structures, the number of resonators on each arm was set to 7. Figure 7(a) shows a microscope picture of the fabricated MZI. We first aligned the single-mode output grating couplers to a fiber array. Next, we aligned a single, cleaved standard fiber at the MMGC input by means of a nanopositioner. We then measured the wavelength response at RE-MZM outputs 1 and 2 for different values of transverse input fiber displacement. Figure 7 shows measured optical power levels at output 1 at wavelengths around the resonance at $\lambda_{\text {res }}=1545 \mathrm{~nm}$. The input fiber position corresponds to lateral displacements between $-10 \mu \mathrm{m}$ and $10 \mu \mathrm{m}$ and the raw data was normalized to the maximum transmission to clearly identify the effect of the resonances. By simultaneously monitoring the power in both outputs at $1545 \mathrm{~nm}$ we found that the structure can be balanced with a transverse displacement of $+3 \mu \mathrm{m}$ at the resonant wavelength (see Figure 7(c)). The measured excess loss due to the alignment (calculated by adding the power in both outputs) as a function of the transverse displacement is represented in Figure 7(d). At the position for balanced outputs (quadrature point) the excess loss is less than $0.1 \mathrm{~dB}$. Moreover, the power imbalance at the centered fiber position $(9 \mathrm{~dB})$ indicates that fabrication asymmetries introduced a phase deviation at $\lambda_{\text {res }}$ on the order of 50 degrees.

On the other hand, for a wavelength far off resonance $(\lambda=1555 \mathrm{~nm})$ the power imbalance at the central position is $-2.5 \mathrm{~dB}$ which corresponds to a phase deviation of just -16 degrees. The discrepancy of the on-resonance and off-resonance phase deviations is the result of an additional phase shift introduced by the resonators as a consequence of a spectral misalignment of the resonances between the arrays coupled to the top and bottom waveguides. By fitting the output spectral responses, we estimated a resonant wavelength misalignment of just $0.15 \mathrm{~nm}$ between the two arrays. By comparison with test structures containing a single resonator array coupled to a single bus waveguide, it was found that the mismatch between the top and bottom arrays was the primary source of resonance wavelengths variation (as opposed to intra-array variations). The mismatch between the arrays can be compensated by rebiasing of the Mach-Zehnder interferometer to the quadrature point.

\section{CONCLUSIONS}

In this work, we have presented the design of resonantly enhanced Mach-Zehnder modulators (RE-MZM) for silicon photonics that aggressively reduce the size and power consumption of conventional MZM approaches based on straight waveguide phase shifters.

On one side, the RF power consumption is reduced by including arrays of cascaded resonators on each arm. The effect of over-coupled resonances allows an enhanced modulation efficiency that reduces the total size and capacitance without excessively penalizing the insertion loss. By optimizing the resonator with a novel design, we achieved a very compact cavity size (down to $3.3 \mu \mathrm{m}$ radius in a smaller design with thicker waveguide and vertical PIN junctions) and thus a significant modulation enhancement in an optical bandwidth larger than $2 \mathrm{~nm}$. Moreover, we have shown the better suitability of a vertical PIN junction design in comparison with a lateral one (resonator design with $5 \mu \mathrm{m}$ radius), since in the former device the wide resonator mode can still have a large overlap with the junction by increasing the size of the embedded diode with a larger overlay between the n-type and p-type implanted layers. The numbers of resonators per arm have been set to maintain a good compromise between insertion loss, OMA and cutoff frequency. The final devices allow considerable reductions in the phase shifter length and RF energy consumption in comparison with a straight waveguide MZM based on equivalent PIN junctions $(\sim 4 \times$ and $\sim 7.7 \times$, respectively for the RE-MZMs with lateral and vertical junctions). The compact size of the RE-MZMs also enables the design of a driving electrode configuration that behaves as a lumped element up to the target data rate of $50 \mathrm{Gbps}$. Experimental results of the fabricated RE-MZM with lateral junction showed that a $3 \mathrm{~dB}$ optical bandwidth of more than $3.8 \mathrm{~nm}$ assures a good modulator performance for a thermal range of operation of more than $50^{\circ} \mathrm{C}$ without the need of any thermal control system.

Furthermore, the phase tuner that is required to correct fabrication asymmetries between the MZM arms has been substituted by an integration scheme in which the quadrature point is passively set during the attachment of the input fiber. The phase relation between the MZM arms is enabled by positioning of the input fiber on top of a Multimode Grating Coupler also used as the first splitter element of the interferometer. An optimized design of the MMGC allows a complete 
correction of any phase deviation with a maximum excess loss of $0.5 \mathrm{~dB}$. Experimental results have shown excellent performance and stability of a proof-of-concept device.

\section{ACKNOWLEDGEMENTS}

This work was supported by the European Research Council under contract number 279770 for the project "Frontiers of Integrated Silicon Nanophotonics in Telecommunications" as well as by the Excellence Initiative of the German federal and state governments.

\section{REFERENCES}

[1] Miller, D.A., "Device requirements for optical interconnects to silicon chips," Proc. IEEE, 97(7), pp. 1166-1185 (2009).

[2] Vlasov, Y.A., "Silicon CMOS-integrated nano-photonics for computer and data communications beyond 100G," IEEE Comm. Mag., 50(2) (2012).

[3] Reed, G.T., Mashanovich, G., Gardes, F.Y. and Thomson, D.J., "Silicon optical modulators," Nat. Phot., 4(8), pp. 518-526 (2010).

[4] Li, Y., Zhang, Y., Zhang, L., and Poon, A.W., "Silicon and hybrid silicon photonic devices for intra-datacenter applications: state of the art and perspectives," Photon. Res., 3(5), pp. B10-B27 (2015).

[5] Xu, Q., Schmidt, B., Pradhan, S. and Lipson, M., "Micrometre-scale silicon electro-optic modulator," Nat., 435(7040), pp. 325-327 (2005).

[6] Li, G., Krishnamoorthy, A.V., Shubin, I., Yao, J., Luo, Y., Thacker, H., Zheng, X., Raj, K., and Cunningham, J.E., "Ring resonator modulators in silicon for interchip photonic links," J. Sel. Top. Quant. Electron., 19(6), pp. 95-113 (2013).

[7] Dong, P., Chen, L., and Chen, Y.K., "High-speed low-voltage single-drive push-pull silicon Mach-Zehnder modulators," Opt. Expr., 20(6), pp. 6163-6169 (2012).

[8] Zheng, X., Chang, E., Amberg, P., Shubin, I., Lexau, J., Liu, F., Thacker, H., Djordjevic, S.S., Lin, S., Luo, Y. and Yao, J., "A high-speed, tunable silicon photonic ring modulator integrated with ultra-efficient active wavelength control," Opt. Expr., 22(10), pp. 12628-12633 (2014).

[9] Romero-Garcia, S., Shen, B., Merget, F., Marzban, B., and Witzens, J., "Alignment tolerant couplers for silicon photonics," J. Sel. Top. Quant. Electron. 21(6), pp. 765-778 (2015).

[10] Akiyama, S., Kurahashi, T., Baba, T., Hatori, N., Usuki, T., and Yamamoto, T., "A 1 V peak-to-peak driven 10Gbps slow-light silicon Mach-Zehnder modulator using cascaded ring resonators," Appl. Phys. Expr., 3(7), Art. ID 072202 (2010).

[11] Akiyama, S., Kurahashi, T., Morito, K., Yamamoto, T., Usuki, T., and Nomura, S., "Cascaded-ring-resonatorloaded Mach-Zehnder modulator for enhanced modulation efficiency in wide optical bandwidth," Opt. Expr., 20(15), pp. 16321-16338 (2012).

[12] Romero-García, S., Moscoso-Mártir, A., Azadeh, S. S., Müller, J., Shen, B., Merget, F., and Witzens, J., "Highspeed resonantly enhanced silicon photonics modulator with a large operating temperature range," Opt. Lett., 42(1), pp. 81-84 (2017).

[13] Romero-Garcia, S., Marzban, B., Merget, F., Shen, B., and Witzens, J., "Edge couplers with relaxed alignment tolerance for pick-and-place hybrid integration of III-V lasers with SOI waveguides," J. Sel. Top. Quant. Electron., 20(4), pp. 369-379 (2014).

[14]Lim, A. E. J., Song, J., Fang, Q., Li, C., Tu, X., Duan, N., Chen, K. K., Tern, R. P. C., and Liow, T. Y., "Review of silicon photonics foundry efforts," J. Sel. Top. Quant. Electron., 20(4), pp. 405-416 (2014).

[15] Spencer, D.T., Bauters, J.F., Heck, M.J., and Bowers, J.E., "Integrated waveguide coupled $\mathrm{Si}_{3} \mathrm{~N}_{4}$ resonators in the ultrahigh-Q regime," Optica, 1(3), pp. 153-157 (2014).

[16] Müller, J., Hauck, J., Shen, B., Romero-García, S., Islamova, E., Azadeh, S.S., Joshi, S., Chimot, N., MoscosoMártir, A., Merget, F., Lelarge, F., and Witzens, J., "Silicon photonics WDM transmitter with single section semiconductor mode-locked laser,” Adv. Opt. Technol., 4(2), pp. 119-145 (2015). 
[17] Azadeh, S. S., Romero-García, S., Merget, F., Moscoso-Mártir, A., von den Driesch, N., Buca, D. and Witzens, J., "Epitaxially grown vertical junction phase shifters for improved modulation efficiency in silicon depletiontype modulators," in Proc. SPIE, 9516, Art. ID 95160T (2015).

[18] Azadeh, S.S., Merget, F., Romero-García, S., Moscoso-Mártir, A., von den Driesch, N., Müller, J., Mantl, S., Buca, D. and Witzens, J., "Low V $\pi$ Silicon photonics modulators with highly linear epitaxially grown phase shifters," Opt. Expr., 23(18), pp. 23526-23550 (2015).

[19]Reed, G.T., Mashanovich, G.Z., Gardes, F.Y., Nedeljkovic, M., Hu, Y., Thomson, D.J., Li, K., Wilson, P.R., Chen, S.W., and Hsu, S.S. "Recent breakthroughs in carrier depletion based silicon optical modulators," Nanophotonics, 3(4-5), pp. 229-245 (2014). 